\title{
Clinical efficacy of upper limb robotic therapy in people with tetraplegia: a pilot randomized controlled trial
}

\author{
Junsik Kim ${ }^{1} \cdot$ Bum Suk Lee ${ }^{1} \cdot$ Hye-Jin Lee ${ }^{1} \cdot$ Hye-Ri Kim ${ }^{1} \cdot$ Duk-Youn Cho $^{2} \cdot$ Jung-Eun Lim $^{2} \cdot$ Jin-Ju Kim $^{1}$. \\ Ha Yeon $\mathrm{Kim}^{2} \cdot$ Zee-A Han $\mathbb{B}^{1}$
}

Received: 21 March 2018 / Revised: 10 July 2018 / Accepted: 23 July 2018 / Published online: 11 September 2018

(c) International Spinal Cord Society 2018

\begin{abstract}
Study design A pilot randomized controlled trial.

Objectives To evaluate the clinical efficacy of upper limb robotic therapy in people with tetraplegia.

Setting Inpatient rehabilitation hospital in Seoul, Korea.

Methods Participants were randomly allocated to a robotic therapy (RT) or occupational therapy (OT) group. Both groups received usual care plus $30 \mathrm{~min}$ of additional therapy per day for 4 weeks. The additional therapy provided to the OT group was OT, and the additional therapy provided to RT group was RT using the Armeo Power. Primary outcomes were the Medical Research Council scale of each key muscle and Upper Extremity Motor Score (UEMS) for the trained arm. Secondary outcomes were the Spinal Cord Independence Measurement version III (SCIM-III) subscale and total score. Evaluations were performed at baseline and 4 weeks.

Results A total of 34 individuals with tetraplegia were included; 17 in each group. At 4 weeks, the median (IQR) change in UEMS in the RT group was $1 / 25$ (0 to 3 ) points compared with $0 / 25(-1$ to 1$)$ points in the OT group $(p=0.03)$. The median (IQR) change in total SCIM-III score in the RT group was 7/100 (1.5 to 11) points compared with 0/100 ( -8 to 4$)$ points in the OT group $(p<0.01)$.

Conclusions There were small improvements in motor strength and SCIM-III scores in the RT group, but there were no statistically significant differences between the groups. Further studies are required for a better understanding of the effects of RT for people with tetraplegia.
\end{abstract}

\section{Introduction}

Spinal cord injury (SCI) leads to the disruption of motor and sensory signals and the autonomic nervous system in the spinal cord [1]. The reported SCI incidence varies between 10.4 and 83 per million per year, and approximately onethird of those who sustain a SCI have cervical injuries

Electronic supplementary material The online version of this article (https://doi.org/10.1038/s41393-018-0190-z) contains supplementary material, which is available to authorized users.

Zee-A Han

zeeahan@catholic.ac.kr

1 Department of Rehabilitation Medicine, National Rehabilitation Center \& Hospital, Seoul, Korea

2 Translational Research Center for Rehabilitation Robots, National Rehabilitation Center \& Hospital, Seoul, Korea resulting in tetraplegia [2]. Individuals with tetraplegia present with impaired upper extremity, trunk, and lower extremity strength and function [1]. Among the impaired functions, upper extremity function is one of the most important goals of rehabilitation for these individuals [3]. Therefore, there have been many efforts to improve upper extremity function through physical therapy (PT), occupational therapy (OT), orthoses, functional electrical stimulation, tendon transfer surgery, and nerve transfer surgery [49]. Conventional PT and OT are the most commonly applied treatments for improving strength and endurance [10]. Although not fully understood, neuroplasticity is thought to be the underlying recovery mechanism behind SCI recovery and higher repetition, intensity, and taskspecific training are believed to lead to better improvements than observed with other interventions [11-13]. However, the quantity of repetition during conventional OT in inpatient rehabilitation hospitals may not be sufficient [14]. Furthermore, the variability of experience between 
therapists may impede effective and homogenous treatment. In this context, robotic therapy (RT) can be an alternative way of delivering rehabilitative therapy to patients with tetraplegia because RT can provide high intensity, high repetition, and task-specific training consistently with less effort required from therapists compared to conventional OT [15].

Various upper extremity rehabilitation robotics (UER) have been developed, and are currently being used in the clinic $[11,12,16-18]$. These UERs can be classified by their different mechanical designs and control strategies. Common mechanical designs include end-effector, exoskeleton, and planar types. The end-effector types attach to the most distal part of the individual's upper extremity only, whereas the exoskeleton type attaches to both proximal and distal segments of the upper extremity (such as the shoulder, elbow, forearm, wrist, and fingers). Thus, the exoskeleton type of UER is safe to apply in weak, non-antigravity muscles because it provides better support than other types of UERs.

Compared to the abundant studies about the application of UER to those who have had a stroke, studies investigating the efficacy of UERs in people with tetraplegia are relatively limited. One case study provided evidence of some improvements in motor performance and spasticity [19], while several other studies provided evidence focusing more on the feasibility of UER rather than the clinical efficacy [15, 20-24]. The goal of this pilot randomized controlled trial was to investigate the efficacy of UER as an adjunctive treatment to conventional OT in people with tetraplegia.

\section{Methods}

\section{Participants}

This study was conducted at a single center (National Rehabilitation Center, Seoul, South Korea) and approved by the institutional review board of the National Rehabilitation Center.

People with tetraplegia who were admitted to the National Rehabilitation Center from January 2015 to April 2016 were screened for recruitment according to the following eligibility criteria. Inclusion criteria were (1) age greater than 19 years, (2) time since injury less than 1 year, (3) upper extremity strength of at least one key muscle was trace or greater, and (4) able to understand instructions. Exclusion criteria were (1) the cause of motor weakness was not due to SCI, (2) severe spasticity as indicated by grade 3 or 4 according to the Modified Ashworth Scale [25], (3) inability to sit in a wheelchair or on a standard chair for more than $30 \mathrm{~min}$, (4) limited passive range of motion as indicated by less than $50 \%$ of the normal range in the shoulder, elbow or wrist joint, and (5) severe shoulder pain that interferes with upper limb therapy. All participants provided a written informed consent before inclusion in the study.

\section{Study design}

This study was a pilot, single-blinded randomized controlled trial in a single rehabilitation hospital. Baseline evaluations were performed by a blinded assessor. Block randomization was used to ensure that motor complete (American Spinal Injury Association (ASIA) Impairment Scale [AIS] A and B) and motor incomplete (AIS C and D) participants were equally allocated to each group (RT and OT groups). Randomization was generated with a computer program (block size of eight was used, no person other than the independent statistician knew the block size or randomization schedule), and the results were concealed in an envelope. After baseline evaluations, the research coordinator contacted the independent statistician to receive the participant's allocation. Participants and their therapists were notified of the participant's allocation. Separate therapists were responsible for delivering the OT and RT therapies. At 4 weeks, participants' outcomes were measured by a blinded assessor who was different from the assessor used for the baseline assessments (for practical reasons). All assessors had at least 5 years' experience in SCI as occupational therapists and were blinded at all time points and were not involved in any therapy.

\section{Intervention}

The RT group received OT with an additional 30 min of RT using the Armeo Power (AP) each day, while the OT group received OT with another additional $30 \mathrm{~min}$ of OT. The side of the body with the lower Upper Extremity Motor Score (UEMS) (according to the International Standards for Neurological Classification of SCI [ISNCSCI]) was selected as the therapy side. If the total score was the same on both sides, the side with a key muscle graded as trace was selected. If all of these were the same, the dominant side was selected as the therapy side.

In the RT group, AP (Hocoma, Switzerland) was used as the robotic intervention. (AP is shown in Fig. 1). The participant's arm is placed so that it rests on the exoskeleton structure. The exoskeleton system provides antigravity weight support, and it also provides assistance or resistance to the participant's movement as needed. It allows six actuated axes of movements (1. shoulder flexion/extension, 2. shoulder adduction/abduction, 3. shoulder internal/ external rotation, 4. elbow flexion/extension, 5. forearm pronation/supination, 6. wrist flexion/extension) with an 


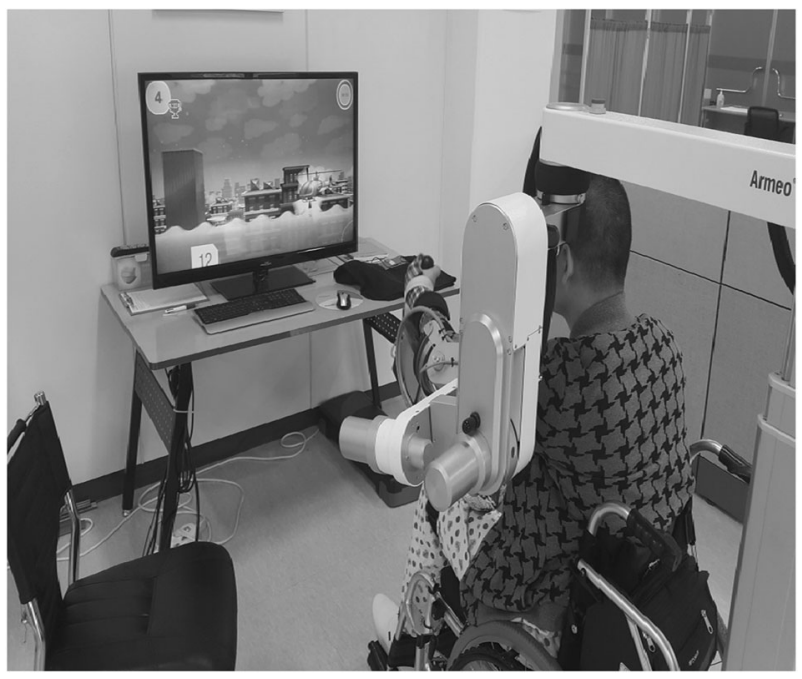

Fig. 1 Participant training with Armeo Power (AP)

additional grip module. Each actuator is located close to the target joints [26]. At the most distal part of the robot, the grip module senses the grip and release of the hand grip. Audio-visual feedback on participant's performance was provided during the treatment. Most programs that were applied during the treatment sessions were "Functional Exercise Programs," which were individualized programs that could target specific joint movements with more intensity and repetition. The UER provided resistance when a participant could move actively during the given task (i.e., farmer [watering a garden], high flyer [controlling flying character's altitude with wrist/shoulder]) but when the participant was unable to complete the task, resistance was removed, and assistance was provided (range of motion exercise). During the 30-min therapy sessions, six different programs (4.8 min per each program) were applied, and between programs, there were about $15 \mathrm{~s}$ of rest for preparation. One hundred to 300 repetitions of a movement were performed, mainly involving the key muscles with a higher potential for recovery (trace, poor, fair or good strength). As strength improved, some of the participants, who mostly needed assistance during the task in the beginning, underwent resistance training (strengthening exercise) at a later point in time. The number of repetitions in each therapy session was also increased as participants' strength improved.

For conventional OT, a licensed occupational therapist with at least 5 years of experience in SCI treatment administered the therapy. During the $30 \mathrm{~min}$ of conventional OT, the main components of therapy were training for activities of daily living (i.e., feeding, personal hygiene, transfer, pressure relief) and strengthening for such activities. Strengthening exercises were done with a dumbbell or resistance was provided by the therapist during movement.
OT also focused on the key muscles with the highest potential for recovery.

All participants also received two conventional PT (30 min) and one OT (30 min) sessions per day. Bedside mobility (i.e., roll-over, sit up, sitting balance training), transfer training (i.e., bed to wheelchair, wheelchair to toilet, wheelchair to car), manual wheelchair propulsion training and strengthening related to these activities were the main components of the PT sessions. There was some overlap (bedside mobility, transfer, pressure relief training) between PT and OT, but OT uniquely provided feeding, personal hygiene, computer, and electric wheelchair manipulation training.

\section{Outcome measures}

\section{Primary outcomes}

Primary outcomes were the Manual Muscle Test (MMT) score of each key muscle comprising the UEMS (as per the ISNCSCI) (i.e., C5: elbow flexors, C6: wrist extensors, C7: elbow extensors, $\mathrm{C} 8$ : finger flexors to the middle finger, and T1: fifth finger abductors), and the total UEMS (sum of each key muscles) for the arm that received the additional therapy. The MMT scores were based on the Medical Research Council (MRC) scale by ASIA guidelines. The evaluation was done at baseline and 4 weeks.

\section{Secondary outcomes}

Secondary outcomes were the scores of each subscale of SCIM-III and the total SCIM-III score. SCIM-III is a reliable way of measuring functional recovery specifically in people with SCI [27]. It is composed of four subscales: (1) Self-Care (six items, score ranges from 0 to 20), (2) Respiration and Sphincter Management (six items, score ranges from 0 to 40), (3) Mobility of Room and Toilet (three items, score ranges from 0 to 10), (4) Mobility Indoors and Outdoors on Even Surface (six items, score ranges from 0 to 40). A detailed description of SCIM-III is provided in Supplementary Table 1. We evaluated the SCIM-III score of each participant at baseline and 4 weeks.

\section{Statistical analysis}

All analyses were conducted using SPSS (IBM Corp. Released in 2011. IBM SPSS Statistics for Windows, Version 20.0. Armonk, NY: IBM Corp.). Participants' baseline characteristics were reported using descriptive statistics. Intention-to-treat analysis was done with multiple imputations for the missing values from the dropouts. We created five multiple imputed data sets with five iterations, using SPSS. Variables imputed were outcome measures at 
4 weeks for the four dropouts. Both primary and secondary outcomes were analyzed using the change in score from baseline to 4 weeks. Mann-Whitney $U$ test was used to compare the changes between the two groups for all data which were not normally distributed. Outcomes were reported as median change (25-75\% percentiles). $P$ values less than 0.05 were considered statistically significant.

\section{Clinical registration}

URL: http://cris.nih.go.kr. Unique identifier: KCT0002745 (registered retrospectively).

\section{Results}

\section{Participant characteristics}

A total of 52 participants with tetraplegia were screened for inclusion, but 12 refused to participate, and six participants did not meet the inclusion criteria (due to pressure ulcer [1 participant] and severe pain [5 participants]). Therefore, 34 participants were randomly allocated to each group (RT $[n=17]$ and OT $[n=17])$. During the study, two participants from each of the two groups discontinued therapy due to personal issues. At 4 weeks, 15 participants remained in each of the two groups. Seventeen were included for intention-to-treat analysis (see Fig. 2 for the CONSORT flowchart). No adverse event related to the intervention, including fatigue, were reported during the entire trial. The demographic characteristics investigated were as follows: sex, age, days after onset of injury, AIS classification, neurological level of injury, baseline MRC, baseline UEMS, and SCIM-III subscale score, and total SCIM-III score. Table 1 summarizes the baseline characteristics of the study participants.

\section{Motor strength changes}

At 4 weeks, the median change in the UEMS (/25 points) for the limb receiving therapy in the RT group was 1 compared with 0 in the OT group $(p=0.03)$. None of the changes in the MRC scale of each key muscle was statistically significant (the details are reported in Table 2, and the individual data set is provided in Supplementary Table 2).

\section{SCIM-III score}

At 4 weeks, the median change in total SCIM-III score in the RT group was 7 compared with 0 in the OT group ( $p$ $<0.01$ ). Among the four subscales of SCIM-III, the median change of mobility (room and toilet) in the RT group was 1 compared with 0 in the OT group $(p=0.02)$. Changes in other subscales were not statistically significant. A full report on the change of SCIM-III score from baseline to 4 weeks are reported in Table 3, and full individual data are also provided in Supplementary Table 2.

\section{Discussion}

Currently, UER therapy is a modality that can provide training with more repetitions at a higher intensity than conventional OT. Some UERs can also provide taskspecific training and can be easily combined with audiovisual feedback. In addition, objective assessments can be done through computer software [15, 28]. In individuals who have had a stroke, the UER showed minor but significant improvements in the Fugl-Meyer Assessment of arm scores and muscle strength, compared to non-robotic therapies [29]. Unlike the abundant research on UER application in the stroke population, the SCI population is a specific group with limited evidence on UER efficacy. Recently, a systematic review showed that previous studies on robot-assisted upper extremity rehabilitation for people with tetraplegia were mostly case series or case studies with small sample sizes [30]. One randomized controlled trial that included UER only investigated the modulatory effects of transcranial direct current stimulation for improving upper extremity function in people with incomplete tetraplegia, and did not focus on UER efficacy [31].

Participants demonstrated motor strength improvements in UEMS (sum of C5 to T1) after a total 20 sessions ([30 min/session] $\times$ five sessions/week $\times$ four weeks) of OT plus RT. Improvements in UEMS were also reported by Francisco GE et al. [32]. In this case series study, the intervention protocol included RT ([three hours/session] $\times$ three sessions/week $\times$ four weeks) in both upper extremities using an exoskeleton type UER (MAHI Exo-II) with five degrees of freedom. The UER used in our study was also an exoskeleton type UER with six degrees of freedom [33, 34]. However, in contrast to the positive results found with UER in motor strength, a case series study by Cortes et al., which included ten people with chronic tetraplegia (time since injury 2-8 years) did not show significant motor strength improvement. In that study, RT (one hour/day $\times$ three times/ week) was provided for six weeks with the InMotion 3.0 Wrist robot (Interactive Motion Technologies, Inc., MA, USA). Compared to our study, participants' time since injury was longer and the UER utilized had only three degrees of freedom [28]. Exoskeleton type UER with multiple degrees of freedom can be a safe option to train multiple joints safely [35]. However, further research is 


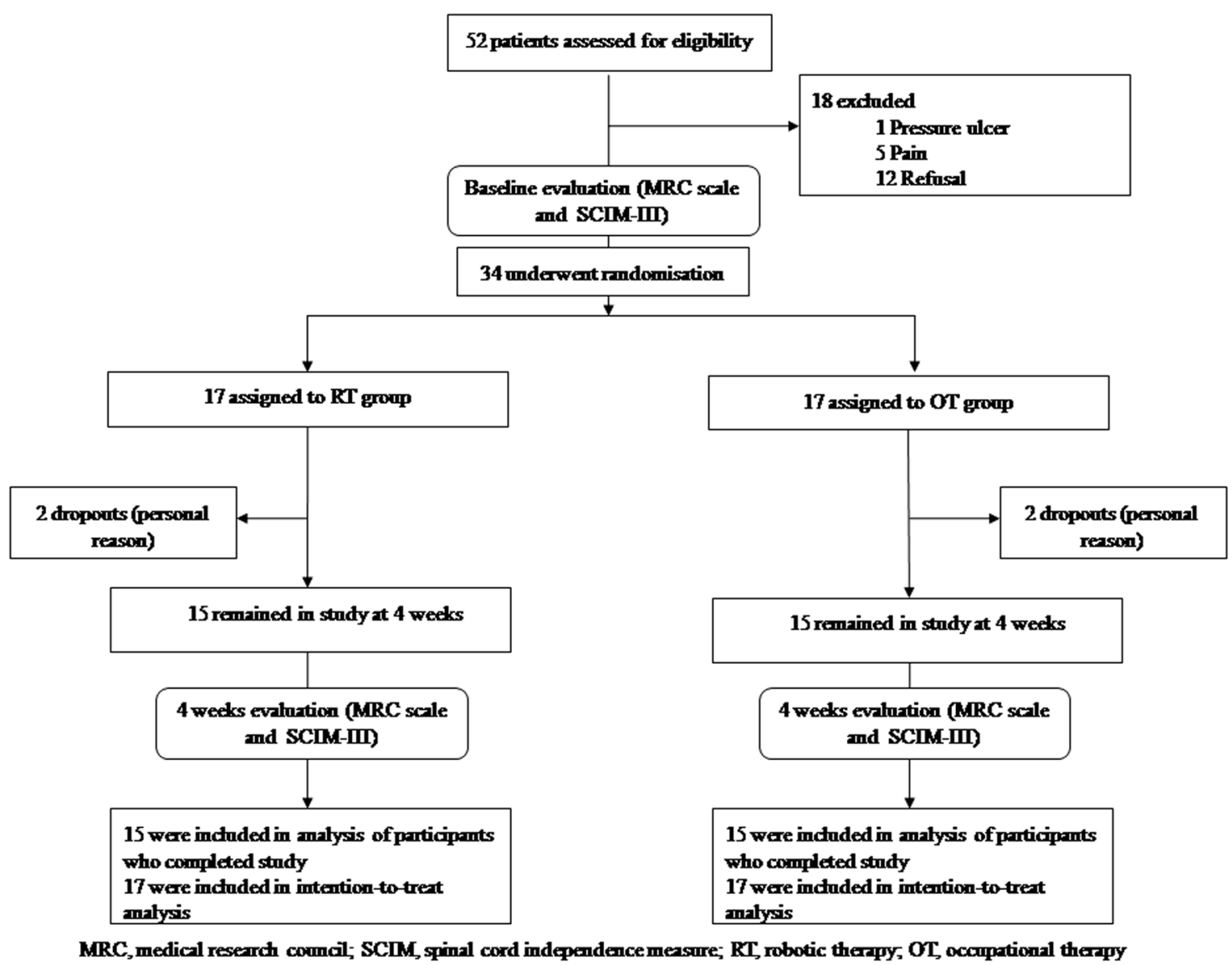

Fig. 2 CONSORT flowchart

needed to determine the most beneficial type of UER for individuals with tetraplegia.

Criteria for selecting the side of the body to treat were different between previous studies. In our study, the therapy side was selected by comparing the UEMS on both sides, and the side with the lower score was selected. If the UEMS was same on both sides, the side with more trace graded muscles was selected. Another study also showed more motor strength improvements in the more affected arm (the side with the lower UEMS) when UER was applied to both upper extremities [28]. After SCI, the potential of strength gains between zero and trace graded muscles varies significantly. A total of 75 to $95 \%$ of trace muscles recovered antigravity strength at 1 year, in contrast to $27 \%$ of zero graded muscles [36]. Therefore, intensive training of trace graded muscles is critical. However, it is difficult to train trace graded muscles properly using conventional OT, and the variability in treatment experience between therapists may also impede the homogeneity of the treatment. Such factors may prevent effective treatment at important periods where neurological improvement is still possible, which makes UER a valuable intervention.
The RT group was provided more repetition to the targeted key muscles than the OT group. Conventional OT provided in the clinical field today is composed mostly of training for activities of daily living according to the patient's neurological level of injury. There can be variability in the number of repetitions and the amount of strength training provided. Lack of sufficient repetitions during conventional OT has been reported previously [14]. Insufficient training may impede individuals from reaching their maximum motor strength recovery. Therefore, there is a need for conventional OT programs to have a sufficient number of repetitions of key muscles and also increase the dosage of strength training provided. People with SCI can also be taught and encouraged to perform exercises unsupervised to increase the quantity of the training. Moreover, using the UER with audio-visual feedback combined with task-specific training also may have acted synergistically to promote recovery through neuroplasticity [15].

With motor strength improvements reported in our study, we expected to see functional improvements in the RT group because increases in strength lead to increases in function [8, 37]. Based on our experience (about 300 
Table 1 Baseline characteristics of the study participants

\begin{tabular}{lll}
\hline Characteristics & $\begin{array}{l}\text { RT Group } \\
(n=17)\end{array}$ & $\begin{array}{l}\text { OT group } \\
(n=17)\end{array}$ \\
\hline Sex: numbers & & \\
Male & 14 & 14 \\
Female & 3 & 3 \\
Age: years (SD) & $56.65 \pm 13.62$ & $47.12 \pm 14.90$ \\
Days after onset: median [IQR] & $93[51.5$ to & $157[88.0$ to \\
& $172.5]$ & $262.5]$ \\
AIS Grade: numbers & & \\
AIS-A & 4 & 4 \\
AIS-B & 3 & 3 \\
AIS-C & 2 & 2 \\
AIS-D & 8 & 8
\end{tabular}

Neurological level of injury: numbers

$\begin{array}{lll}\mathrm{C} 2 & 1 & 1 \\ \mathrm{C} 3 & 1 & 1 \\ \mathrm{C} 4 & 7 & 8 \\ \mathrm{C} 5 & 4 & 4 \\ \mathrm{C} 6 & 2 & 2 \\ \mathrm{C} 7 & 1 & 1 \\ \mathrm{C} 8 & 1 & 0\end{array}$

Baseline MRC score of key muscles (/5 pts):median [IQR]

$\begin{array}{lll}\text { C5: Elbow flexors } & 3[2.5 \text { to } 3] & 3[2.5 \text { to } 3] \\ \text { C6: Wrist extensors } & 3[1.5 \text { to } 3] & 3[1.5 \text { to } 3] \\ \text { C7: Elbow extensors } & 3[2 \text { to } 3] & 3[2 \text { to } 3] \\ \text { C8: Finger flexors } & 2[0.5 \text { to } 3] & 1[0 \text { to } 2] \\ \text { T1: 5th finger abductors } & 2[0 \text { to } 2.5] & 0[0 \text { to } 1.5] \\ \text { Baseline UEMS /25 pts (Sum of C5-T1): median [IQR] }\end{array}$

13 [8 to 15$] \quad 10$ [6.5 to 14$]$

Baseline SCIM-III subscale score: median [IQR]

\begin{tabular}{|c|c|c|}
\hline I. Self-care (/20 pts) & $4[1.5$ to 6$]$ & $4[0.5$ to 5.5$]$ \\
\hline $\begin{array}{l}\text { II. Respiration \& Sphincter (/40 } \\
\text { pts) }\end{array}$ & $20[15$ to 33.5$]$ & 21 [15 to 32$]$ \\
\hline $\begin{array}{l}\text { III. Mobility: room \& toilet (/10 } \\
\text { pts) }\end{array}$ & $2[0$ to 7.5$]$ & 4 [1 to 7$]$ \\
\hline $\begin{array}{l}\text { IV. Mobility: indoor \& outdoor } \\
\text { (/30 pts) }\end{array}$ & $4[0.5$ to 16$]$ & $2[0$ to 6$]$ \\
\hline $\begin{array}{l}\text { Baseline total SCIM score (/100 } \\
\text { pts) }\end{array}$ & 24 [19 to 63.5$]$ & $\begin{array}{l}27[22.5 \text { to } \\
50.5]\end{array}$ \\
\hline
\end{tabular}

$R T$ robotic therapy, $O T$ occupational therapy, $S D$ standard deviation, $I Q R$ interquartile range, AIS American Spinal Cord Injury Association Impairment Scale, MRC medical research council, SCIM-III Spinal Cord Independence Measurement Version III, UEMS Upper Extremity Motor Score

individuals with SCI admitted per year) we considered any minimal strength gain to be clinically significant and predicted that such minimal gains would transfer into an improved function. For instance, a slight improvement in upper extremity strength may enhance the feasibility of a person with tetraplegia to manipulate an electric wheelchair
Table 2 Primary outcomes

Intention-to-treat analysis with imputed data $(N=34)$

\begin{tabular}{|c|c|c|c|}
\hline & \multicolumn{2}{|c|}{ Median change $[I Q R]$} & \multirow[b]{2}{*}{$P^{*}$} \\
\hline & $R T(n=17)$ & $O T(n=17)$ & \\
\hline \multicolumn{4}{|c|}{ MRC scale of key muscle at trained side } \\
\hline C5: Elbow flexors & $0[0$ to 1$]$ & $0[-0.82$ to 0$]$ & 0.21 \\
\hline C6: Wrist extensors & $0[0$ to 1$]$ & $0[-0.5$ to 0$]$ & 0.08 \\
\hline C7: Elbow extensors & $0[0$ to 1$]$ & $0[0$ to 0$]$ & 0.16 \\
\hline C8: Finger flexors & $0[0$ to 1$]$ & $0[0$ to 0.5$]$ & 0.66 \\
\hline T1: 5th finger abductors & $0[0$ to 1$]$ & $0[0$ to 0.08$]$ & 0.59 \\
\hline UEMS at trained side $(/ 25 \mathrm{pts})$ & $1[0$ to 3$]$ & $0[-1$ to 1$]$ & 0.03 \\
\hline \multicolumn{4}{|c|}{ Participants who completed study $(N=30)$} \\
\hline & $R T(n=15)$ & $O T(n=15)$ & $P^{*}$ \\
\hline C5: Elbow flexors & $0[0$ to 1$]$ & $0[0$ to 0$]$ & 0.12 \\
\hline C6: Wrist extensors & $0[0$ to 1$]$ & $0[0$ to 0$]$ & 0.06 \\
\hline C7: Elbow extensors & $0[0$ to 1$]$ & $0[0$ to 0$]$ & 0.08 \\
\hline C8: Finger flexors & $0[0$ to 1$]$ & $0[0$ to 0$]$ & 0.29 \\
\hline T1: 5th finger abductors & $0[0$ to 1$]$ & $0[0$ to 0$]$ & 0.27 \\
\hline UEMS at trained side (/25 pts) & $1[0$ to 3$]$ & $0[-1$ to 1$]$ & 0.02 \\
\hline
\end{tabular}

$I Q R$ interquartile range, $M R C$ medical research council, $R T$ robotic therapy group, $O T$ occupational therapy group, UEMS Upper Extremity Motor Score

*Mann-Whitney $U$ test

or a computer. We initially expected to see improvements in the self-care subscale. However, the mobility (room and toilet) subscale and the total score of SCIM-III were the two outcomes that showed higher scores in the RT group than in the OT group. We hypothesized that improvement in motor strength might have led to a better ability to prevent pressure ulcers, improved electrical wheelchair manipulation, and improved short distance mobility with better support from an assistive device (including walker, crutch, and cane). Finally, the higher total SCIM-III score improvement in the RT group may be the result of the reinforcing effect of the previously mentioned factors. However, measurement by MMT and comparing each item of SCIM-III had questionable reliability and should remain as a hypothesis at this moment. Unfortunately, eight participants showed a small decline in MRC score in one or more key muscles. Nerve entrapment, posttraumatic syringomyelia, and late compression of the spinal cord nerve roots (caused by progressive spondylosis, spinal stenosis, intervertebral disk herniations, and posttraumatic changes) are possible causes of a decrease in motor or sensory function of people with SCI [38]. No pain or fatigue were noted during this study, and the cause for the MRC decline was not identified. Another possible reason for the detected motor decline is that even though the inter-rater reliability of the MMT is good, a disagreement between assessors may have been 
Table 3 Secondary outcomes

\begin{tabular}{|c|c|c|c|}
\hline & \multicolumn{2}{|c|}{ Median change [IQR] } & \multirow[b]{2}{*}{$P^{*}$} \\
\hline & $R T(n=17)$ & $O T(n=17)$ & \\
\hline \multicolumn{4}{|l|}{ SCIM-III subscale } \\
\hline 1. Self care (/20 pts) & $1[0$ to 1.5$]$ & $0[-1.7$ to 1.5$]$ & 0.38 \\
\hline 2. Respiration \& Sphincter (/40 pts) & $0[-1$ to 3$]$ & $0[-6.5$ to 0$]$ & 0.11 \\
\hline 3. Mobility: room \& toilet (/10 pts) & $1[0$ to 3$]$ & $0[-1$ to 1$]$ & 0.02 \\
\hline 4. Mobility: indoor \& outdoor (/30 pts) & $3[0.5$ to 6.5$]$ & $1[0$ to 3.5$]$ & 0.16 \\
\hline Total SCIM-III score (/100 pts) & $7[1.5$ to 11$]$ & $0[-8$ to 4$]$ & $<0.01$ \\
\hline \multicolumn{4}{|l|}{ Participants who completed study $(N=30)$} \\
\hline & $R T(n=15)$ & $O T(n=15)$ & $P^{*}$ \\
\hline 1. Self care (/20 pts) & $0[0$ to 1$]$ & $1[-1$ to 2$]$ & 0.87 \\
\hline 2. Respiration \& Sphincter (/40 pts) & $0[0$ to 3$]$ & $0[-2$ to 0$]$ & 0.19 \\
\hline 3. Mobility: room \& toilet (/10 pts) & $1[0$ to 2$]$ & $0[-1$ to 0$]$ & 0.05 \\
\hline 4. Mobility: indoor \& outdoor (/30 pts) & $2[0$ to 8$]$ & $0[0$ to 3$]$ & 0.07 \\
\hline Total SCIM-III score (/100 pts) & $7[2$ to 11$]$ & $0[-4$ to 4$]$ & $<0.01$ \\
\hline
\end{tabular}

$I Q R$ interquartile range, SCIM-III Spinal Cord Independence Measurement version III, $R T$ robotic therapy group, $O T$ occupational therapy group

*Mann-Whitney $U$ test possible [39, 40]. However, the MMT was conducted by blinded assessors in this study and any noise would have been equally distributed across both groups without systematic bias. There were other limitations to this study. First, this was a single-center study with one specific UER applied. Comparison between different types of UERs should be evaluated in future studies. Second, long-term follow-up was not included. Therefore, the maintenance of effect was not proven. Third, the robot in this study did not provide fine hand movement training. AP did sense the grip strength and required participants to grab the hand module at a certain level of strength and release during the given task. However, it is questionable whether it provided sufficient hand movement training. Fourth, the clinical meaningfulness should be supported by studies with specific assessment tools (ARAT [Action Research Arm Test], Van Leshout test, and GRASSP [Graded and Redefined Assessment of Strength, Sensibility, and Prehension]) other than the MMT. Fifth, spontaneous motor recovery is most significant within the first three months, and this may have caused noise. Even though randomization was performed, due to the small sample size, the number of participants fewer than 100 days since the injury was larger (10 vs. 4) in the RT group. Therefore, a randomization study with a larger sample size is necessary. Finally, the statistician performing the analysis was not blinded raising a possibility of experimenter bias. If future studies can overcome the limitations mentioned above, we believe more meaningful information can be provided.

\section{Conclusions}

There were some improvements in motor strength and functional independence in the RT group but there were no statistically significant differences between the two groups. This may, however, just reflect the small sample size. Superiority over conventional OT cannot be concluded in this study. Large studies are now needed for a better understanding of the effect of RT in people with tetraplegia.

Authors' contributions J.S.K. and Z.A.H. were responsible for designing the study, and were also responsible for writing the protocol and report, extracting and analyzing data, interpreting results, updating reference lists and creating tables and figures. D.Y.C. conducted analyses and extracted and analyzed data and interpreted the results. J. E.L., J.J.K., and H.Y.K. contributed to measuring and collecting data. B.S.L., H.R.K., and H.J.L. provided feedback on the report.

Funding This study was supported by a grant (NRCTR-IN15001) of the Translational Research Center for Rehabilitation Robots, Korea National Rehabilitation Center, Ministry of Health \& Welfare, Korea.

\section{Compliance with ethical standards}

Statement of ethics We certify that all applicable institutional and governmental regulations concerning the ethical use of human volunteers were followed during the course of this research.

Conflict of interest The authors declare that they have no conflict of interest. 


\section{References}

1. Kirshblum SC, Burns SP, Biering-Sorensen F, Donovan W, Graves DE, Jha A. et al. International standards for neurological classification of spinal cord injury (Revised 2011). J Spinal Cord Med. 2011;34:535-46.

2. Wyndaele M, Wyndaele JJ. Incidence, prevalence and epidemiology of spinal cord injury: what learns a worldwide literature survey? Spinal Cord. 2006;44:523-9.

3. Simpson LA, Eng JJ, Hsieh JT, Wolfe DL. The health and life priorities of individuals with spinal cord injury: a systematic review. J Neurotrauma. 2012;29:1548-55.

4. Dunn JA, Sinnott KA, Rothwell AG, Mohammed KD, Simcock JW. Tendon transfer surgery for people with tetraplegia: an overview. Arch Phys Med Rehabil. 2016;97(6 Suppl):S75-80.

5. Fox IK, Davidge KM, Novak CB, Hoben G, Kahn LC, Juknis N, et al. Nerve transfers to restore upper extremity function in cervical spinal cord injury: update and preliminary outcomes. Plast Reconstr Surg. 2015;136:780-92.

6. Hokken W, Kalkman S, Blanken WC, van Asbeck FW. A dynamic pronation orthosis for the C6 tetraplegic arm. Arch Phys Med Rehabil. 1993;74:104-5.

7. Lu X, Battistuzzo CR, Zoghi M, Galea MP. Effects of training on upper limb function after cervical spinal cord injury: a systematic review. Clin Rehabil. 2015;29:3-13.

8. Kloosterman MG, Snoek GJ, Jannink MJ. Systematic review of the effects of exercise therapy on the upper extremity of patients with spinal-cord injury. Spinal Cord. 2009;47:196-203.

9. Harvey LA, Lin CW, Glinsky JV, De Wolf A. The effectiveness of physical interventions for people with spinal cord injuries: a systematic review. Spinal Cord. 2009;47:184-95.

10. Foy T, Perritt G, Thimmaiah D, Heisler L, Offutt JL, Cantoni K, et al. Occupational therapy treatment time during inpatient spinal cord injury rehabilitation. J Spinal Cord Med. 2011;34:162-75.

11. Behrman AL, Bowden MG, Nair PM. Neuroplasticity after spinal cord injury and training: an emerging paradigm shift in rehabilitation and walking recovery. Phys Ther. 2006;86:1406-25.

12. Lynskey JV, Belanger A, Jung R. Activity-dependent plasticity in spinal cord injury. J Rehabil Res Dev. 2008;45:229-40.

13. Sandrine SB, Jean-Rene C. Activity-dependent synaptic plasticity and metaplasticity in spinal motor networks. CurrPharm Des. 2013;19:4498-508.

14. Zbogar D, Eng JJ, Miller WC, Krassioukov AV, Verrier MC. Movement repetitions in physical and occupational therapy during spinal cord injury rehabilitation. Spinal Cord. 2016;55:172.

15. Zariffa J, Kapadia N, Kramer JL, Taylor P, Alizadeh-Meghrazi M, Zivanovic V, et al. Feasibility and efficacy of upper limb robotic rehabilitation in a subacute cervical spinal cord injury population. Spinal Cord. 2012;50:220-6.

16. Raineteau O, Schwab ME. Plasticity of motor systems after incomplete spinal cord injury. Nat Rev Neurosci. 2001;2:263-73.

17. Di Rienzo F, Guillot A, Mateo S, Daligault S, Delpuech C, Rode $\mathrm{G}$, et al. Neuroplasticity of prehensile neural networks after quadriplegia. Neuroscience. 2014;274:82-92.

18. Onifer SM, Smith GM, Fouad K. Plasticity after spinal cord injury: relevance to recovery and approaches to facilitate it. Neurotherapeutics. 2011;8:283-93.

19. Kadivar Z, Sullivan JL, Eng DP, Pehlivan AU, O'Malley MK, Yozbatiran N, et al. Robotic training and kinematic analysis of arm and hand after incomplete spinal cord injury: a case study. IEEE Int Conf Rehabil Robot. 2011;2011:5975429.

20. Cortes M, Elder J, Rykman A, Murray L, Avedissian M, Stampas A, et al. Improved motor performance in chronic spinal cord injury following upper-limb robotic training. NeuroRehabilitation. 2013;33:57-65.
21. Rudhe C, Albisser U, Starkey ML, Curt A, Bolliger M. Reliability of movement workspace measurements in a passive arm orthosis used in spinal cord injury rehabilitation. J Neuroeng Rehabil. 2012;9:37.

22. Zariffa J, Kapadia N, Kramer JL, Taylor P, Alizadeh-Meghrazi M, Zivanovic V, et al. Effect of a robotic rehabilitation device on upper limb function in a sub-acute cervical spinal cord injury population. IEEE Int Conf Rehabil Robot. 2011;2011:5975400.

23. Zariffa J, Kapadia N, Kramer JL, Taylor P, Alizadeh-Meghrazi M, Zivanovic V, et al. Relationship between clinical assessments of function and measurements from an upper-limb robotic rehabilitation device in cervical spinal cord injury. IEEE Trans Neural Syst Rehabil Eng. 2012;20:341-50.

24. Vanmulken DA, Spooren AI, Bongers HM, Seelen HA. Robotassisted task-oriented upper extremity skill training in cervical spinal cord injury: a feasibility study. Spinal Cord. 2015;53:547-51.

25. Meseguer-Henarejos AB, Sanchez-Meca J, Lopez-Pina JA, Carles-Hernandez R Inter- and intra-rater reliability of the Modified Ashworth Scale: a systematic review and meta-analysis. Eur J Phys Rehabil Med. 2017;54:576-90.

26. Maciejasz P, Eschweiler J, Gerlach-Hahn K, Jansen-Troy A, Leonhardt S. A survey on robotic devices for upper limb rehabilitation. J Neuroeng Rehabil. 2014;11:3.

27. Anderson K, Aito S, Atkins M, Biering-Sørensen F, Charlifue S, et al. Functional recovery measures for spinal cord injury: an evidence-based review for clinical practice and research. J Spinal Cord Med. 2008;31:133-44.

28. Frullo JM, Elinger J, Pehlivan AU, Fitle K, Nedley K, Francisco $\mathrm{GE}$, et al. Effects of assist-as-needed upper extremity robotic therapy after incomplete spinal cord injury: aparallel-group controlled trial. Front Neurorobot. 2017;11:26.

29. Veerbeek JM, Langbroek-Amersfoort AC. Wegen EEHv, Meskers CGM, Kwakkel G. Effects of robot-assisted therapy for the upper limb after stroke:a systematic review and meta-analysis. Neurorehabil Neural Repair. 2017;31:107-21.

30. Singh H, Unger J, Zariffa J, Pakosh M, Jaglal S, Craven BC, et al. Robot-assisted upper extremity rehabilitation for cervical spinal cord injuries: a systematic scoping review. Disabil Rehabil Assist Technol 2018:1-12.

31. Yozbatiran N, Keser Z, Davis M, Stampas A, O’Malley MK, Cooper-Hay $\mathrm{C}$, et al. Transcranial direct current stimulation (tDCS) of the primary motor cortex and robot-assisted arm training in chronic incomplete cervical spinal cord injury: a proof of concept sham-randomized clinical study. NeuroRehabilitation. 2016;39:401-11.

32. Francisco GE, Yozbatiran N, Berliner J, O’Malley MK, Pehlivan $\mathrm{AU}$, Kadivar Z, et al. Robot-assisted training of arm and hand movement shows functional improvements for incomplete cervical spinal cord injury. Am J Phys Med Rehabil. 2017;96(10Suppl 1):S171-s7.

33. Calabro RS, Russo M, Naro A, Milardi D, Balletta T, Leo A, et al. Who may benefit from Armeo Power treatment? A neurophysiological approach to predict neurorehabilitation outcomes. PM R. 2016;8:971-8.

34. Nef T, Guidali M, Klamroth-Marganska V, Riener R (2009) ARMin-Exoskeleton robot for stroke rehabilitation. In: Dössel O, Schlegel WC, editors. World Congress on Medical Physics and Biomedical Engineering, September 7-12, 2009, Munich, Germany. IFMBE Proceedings, vol 25/9. Springer, Berlin.

35. Pehlivan AU, Sergi F, Erwin A, Yozbatiran N, Francisco GE, O'Malley MK. Design and validation of the RiceWrist-S exoskeleton for robotic rehabilitation after incomplete spinal cord injury. Robotica. 2014;32:1415-31. 
36. Waters RL, Adkins RH, Yakura JS, Sie I. Motor and sensory recovery following complete tetraplegia. Arch Phys Med Rehabil. 1993;74:242-7.

37. Catz A, Greenberg E, Itzkovich M, Bluvshtein V, Ronen J, Gelernter I. A new instrument for outcome assessment in rehabilitation medicine: Spinal cord injury ability realization measurement index. Arch Phys Med Rehabil. 2004;85:399-404.

38. Little JWBS. Neuromusculoskeletal complications of spinal cord injury. In: Kirshblum S, Campagnolo DI, DeLisa JA (editors).
Spinal cord medicine. Philadelphia: Lippincott Williams \& Wilkins; 2002. p. 241-52.

39. Noreau L, Vachon J. Comparison of three methods to assess muscular strength in individuals with spinal cord injury. Spinal Cord. 1998;36:716.

40. Savic G, Bergström EMK, Frankel HL, Jamous MA, Jones PW. Inter-rater reliability of motor and sensory examinations performed according to American Spinal Injury Association standards. Spinal Cord. 2007;45:444. 